\title{
On Problems, Requirements and Solution Approaches when Supporting Knowledge Intensive Processes in Industry
}

\author{
Christian Lütke Entrup and Thomas Barth \\ Information Systems Institute, University of Siegen, Hölderlinstr. 3, Siegen, Germany, \\ \{luetke-entrup|barth\}@fb5.uni-siegen.de
}

\begin{abstract}
Optimizing and hence reorganizing processes as well as increasing their flexibility and agility are constant challenges companies face in the presence of revolving markets. The term 'Business-Process-Reengineering' (BPR) describes the approach of organizing processes along the customer's requirements. Since those requirements are constantly rising in terms of a product's quality and complexity under simultaneously cost and time pressure, effective and efficient re-use of an organizations accumulated knowledge is seen as an important - if not the only - comparative advantage in developed countries where labor, energy, etc. is of substantially higher cost compared to others. As a consequence, importance as well as intensity of knowledge needed to fulfill an organization's most important processes has risen significantly. This article focuses on providing support of knowledge intensive processes by analyzing product data. Retrieving the relevant knowledge in the context of a given process needs tools and methods beyond the well-known approaches for data or document management or organizational knowledge management. The domain of automotive supplier industry as an example is analyzed with respect to dominant strategic challenges like short lifecycles, complex systems, and collaboration with competitors, to retrieve associated knowledge-related documents, and this way offering opportunities to manage those challenges.
\end{abstract}

Keywords: Knowledge intensive process, Knowledge management, Product data analysis, Similarity search

\section{Introduction}

The global and increasingly all sectors of industry concerning change from seller's to buyer's markets (Tietze 2003) in conjunction with worldwide simultaneously progressing severe competition confronts enterprises of the manufacturing sector, 
especially contract manufacturers, with the problem of rising pressure in cost and competition, whilst seeing the necessity of designing and producing innovative and highly sophisticated products in steadily shortening development cycles. Customers and competitors use all possibilities, e.g. the internet, of gathering knowledge about products to get a quick, independent and comprehensive survey of the market. This way, competitors - even from emerging nations - are able to reduce the technological gap to technologically leading enterprises in less time and cost as some years ago. Customers use the ability of immediate comparison; therefore enterprises need to enhance customer retention by improving surplus values and services around their products. Overall, enterprises have to focus on their customers to fulfill their needs.

Since the 1990s these challenges are answered with the reorientation of the company's organization and culture (Hammer and Champy 2001). The keyword "Business-Process-Reengineering" (BPR) describes the approach of consequently organizing high-merit-processes along the customer's requirements. To meet these requirements, high capabilities of innovation as well as short reaction- and development-cycles are necessary. One important feature of innovation- and development-processes is the high intensity of knowledge (Remus 2002), whereby knowledge becomes the decisive factor in the markets (Jänig 2004). Therefore, knowledge management (KM) and explicit support of knowledge intensive processes can be regarded as crucial and essential for the company's positioning and even continuity in the market.

Common approaches of KM focus on the collection, representation, and distribution of a worker's knowledge. They rely on the ability and willingness of the knowledge owners to explain and share their skills in a certain field. To support the externalization of knowledge, IT-systems have been built that provide functionality for gathering, sorting, and representing one's knowledge in the way of document management. Although these systems evolved and became more powerful, the main problem still remains. The inherent weakness of these approaches is the human factor: Socio-cultural reasons cause cognitive and motivational barriers that interfere with the transfer of knowledge (Zelewski 2005). Furthermore, the already mentioned time pressure prevents knowledge workers from spending additional time on document management and even a motivated expert cannot always couch why precisely this way of doing leads to the desired result.

The approach of supporting knowledge intensive processes described in this article differs from this traditional 'organizational' KM as mentioned above in a way that it does not rely on the explicit externalization of knowledge by the owner, but instead relies on taking advantage of the documents and data generated regularly while working on the subject. The main assumption of this approach is the absence of knowledge in data. According to Riempp (2005), only data is stored in databases, the extraction and recombining to knowledge has to be done by a domain's expert. While working on a subject, the expert uses his expertise to produce a certain output that is highly dependent on his experience. E.g., engineers in the automotive sector use their specific knowledge and experience to design parts 
and components of a new car. The output of this process (besides others) is a 3DCAD-model specifying the geometry of a part, which in turn can be used as input for requests to their suppliers having to produce it. If an expert of the domain who was not involved in the developing process examines the model he will understand e.g. why a part's geometry was designed in a certain way, a certain material was specified etc. because of his own domain knowledge.

Following the assumption that domain knowledge manifests in the documents and data generated in knowledge-intensive processes, a way has to be found to retrieve the adequate documents in a distinct situation and present it to the expert who has to recombine it to knowledge and hereby maybe reuses it independently from its originator. In order to support processes this way a search engine is needed, exceeding the text based search functions of conventional (product-)data management systems significantly. By applying a "fuzzy" search on product data, also to a certain degree similar and not necessarily identical objects can be found whose associated documents provide support in complex decision-making situations being the essential part of knowledge intensive processes.

In this paper, requirements for a framework comprising a general approach as well as methods to support knowledge-intensive processes are discussed. Efficient reuse of knowledge seamlessly within processes is identified as a generic method for support. As one approach to supporting knowledge intensive tasks - which already proved to be successful in industrial scenarios - a similarity analysis is discussed that retrieves "similar" (according to domain specific similarity metrics) documents in a company's data store on the basis of a source document. Beyond these search capabilities, extending the search towards considering more complex knowledge structures in distributed scenarios is also outlined.

The next chapter outlines the methodical approach we applied to conduct the research work utilizing interviews, modeling, prototype implementation/evaluation, and workshops. Chapter 3 describes the results of the performed domain analysis in automotive supplier industry, how it constitutes in the global competition and what kind of problems emerge from the new environment they act in. Subsequent sections show the solutions and approaches to handle these problems, as well as an example of an implementation supporting processes in research and development within an automotive suppliers R\&D department.

\section{Research Method}

The approach applied within the project being the basis of this contribution consists mainly of the following components: Interviews, data and knowledge models, prototype implementations, reviews, and workshops.

The proposed process model for supporting knowledge-intensive processes (s. (Lütke Entrup et al. 2006)) was used as a guideline for the selection of interview partners and the topics for the interviews. Since the processes to be supported act 
as the guideline for the analyses of relevant knowledge and its data representation, interview partners from the involved organizational units were selected. Hence, two persons from conceptual planning, controlling, and R\&D were interviewed and the requirements were compiled from their answers. All interviews were performed going collaboratively through real-life examples from the domain expert's daily work to get a detailed understanding of their approach to solve the sophisticated problems involved in their individual contribution to the overall decisionmaking process. This understanding was subsequently rendered to knowledge and data models as a basis for the design and implementation of adequate tools. Aspects covered by observing the expert's role and activities in the processes comprised the information they need access to (e.g. technical drawings, bills of material, transportation cost) and the technical data sources the information is provided from (e.g. CAD systems, ERP systems) as well. Prototypical implementations of this tool were frequently presented to all the interviewed process participants being with the future key users. The results of these reviews were integrated into the next-generation prototypes and the prototyping cycle was restarted. In concluding workshops the key users were trained at the system integrated to the company's IT infrastructure.

\section{Domain Analysis in Automotive Supplier Industry}

The domain of automotive suppliers experienced dramatic change during the last decades. The customer's rising requirements in matters of cost, quality, and configuration are passed from manufacturers to suppliers. This way, suppliers are forced to design, manufacture and deliver highly innovative, highly complex and high quality products in less time to market, while being confronted with increasing pressure in cost and by an increasing number of competitors in their market.

Automobile manufacturers are increasing the number of outsourced components reducing the number of suppliers at the same time. Hence, suppliers are becoming more and more providers for complete subsystems, thus changing from single-part-supplier to system-developers. These sub-systems or assemblies consist of up to some hundred parts, maybe from different engineering domains, e.g. from metal forming, electrics and electronics exceeding the competencies of most suppliers being typically experts in only one of these domains. This complexity fosters cross-organizational collaboration with competitors and/or subcontractors to complement own competencies to assure continuity in the market.

The major requirements that have to be addressed are therefore

- steady shortening development and production time

- rising complexity of products

- collaboration with competitors 
These requirements affect the IT support of knowledge-intensive processes

- provision of methods to support efficient reuse of knowledge seamlessly within processes (identification of relevant processes, relevant knowledge and adequate models for both)

- provision of tools to support human experts in complex and knowledgeintensive processes (searching/finding/reusing documents and data sets representing relevant process-specific knowledge)

- support processes in distributed environments (decoupling between search/find/reuse and - maybe multiple - knowledge bases)

The aforementioned requirements for a framework to support knowledgeintensive processes comprises methodological as well as tool support to bridge the gap between the actual processes, the human process participants, their knowledge and the actual tools supporting them. To validate this approach, knowledgeintensive processes in automotive and automotive supplier industry were analyzed in the course of projects to design, implement and introduce software tools to support knowledge-intensive processes especially in the early phases of the product lifecycle. A qualitative analysis of the domain was done by interviews with domain- and IT-experts of an automotive supplier. It was derived from these interviews that the development time a new part can be reduced by up $90 \%$ if a template exists which structure is very similar to the one of the new part and that could be slightly modified to obtain the desired new structure. Fig. 1 shows typical questions arising during the work on a product in its lifecycle. The questions aim at the availability of similar previous products that can be included in the process as a template and can be used as a knowledge source. The interviews also elaborated the importance of documents associated with the template part to regain the knowledge used in that context.

The main problem is that there is currently no IT system available which is capable of searching, analyzing, and retrieving those templates on the basis of arbitrary input documents. Available IT systems (namely Product Data Management (PDM) and Product Lifecycle Management (PLM) systems) are almost solely focused on document management aspects like processing and transforming different CAD file formats, versioning of documents etc. The important aspect of managing the knowledge contained in these documents is not addressed by software vendors, at most providing search capabilities using regular expressions for analyzing mainly alphanumerical/text data and starting to offer search in CAD data mostly unrelated to the associated alphanumerical product data.

Hence, experts have to rely on their own or their colleague's commemoration to find a similar product by looking through technical drawings and typically browsing through various file systems containing CAD documents and other files. Having to work on lots of different projects during a year, the chance to remember the right product at the right time is quite low. In the case of a typical small to medium sized enterprise (SME) from automotive supplier industry, several hundred up to thousands of customer requests must be processed each year every one com- 
prising large data sets in maybe many (in the worst case up to one hundred) versions created during one knowledge-intensive core business process.

The rising complexity of products is becoming a major problem for system developers. A new system has to be split in its single parts, every part has to be developed, its actual cost must be estimated, physically manufactured, and finally all parts have to be assembled and delivered. From the very beginning of the development to the final assembly, numerous change requests from the customer have to be regarded. A request as well as a change requests normally consists of a single CAD-file that has to be analyzed by experts from the company with respect to changes since the last version. Analyzing a CAD-file is a very tedious and errorprone process. In a system of about 100 parts a minor change in one of those parts can easily be missed. But the miss of a minor change can have major impact on the following processes: Tools do not fit anymore, parts do not match, etc. The adjustment of those misses is expensive and time consuming. Support can be offered through knowledge-related documents of previous projects that can be found by a similarity analysis on the basis of the part in question and its sub-parts. To analyze a complex product structure approaches of ontology and recursive structure analysis are taken into consideration.

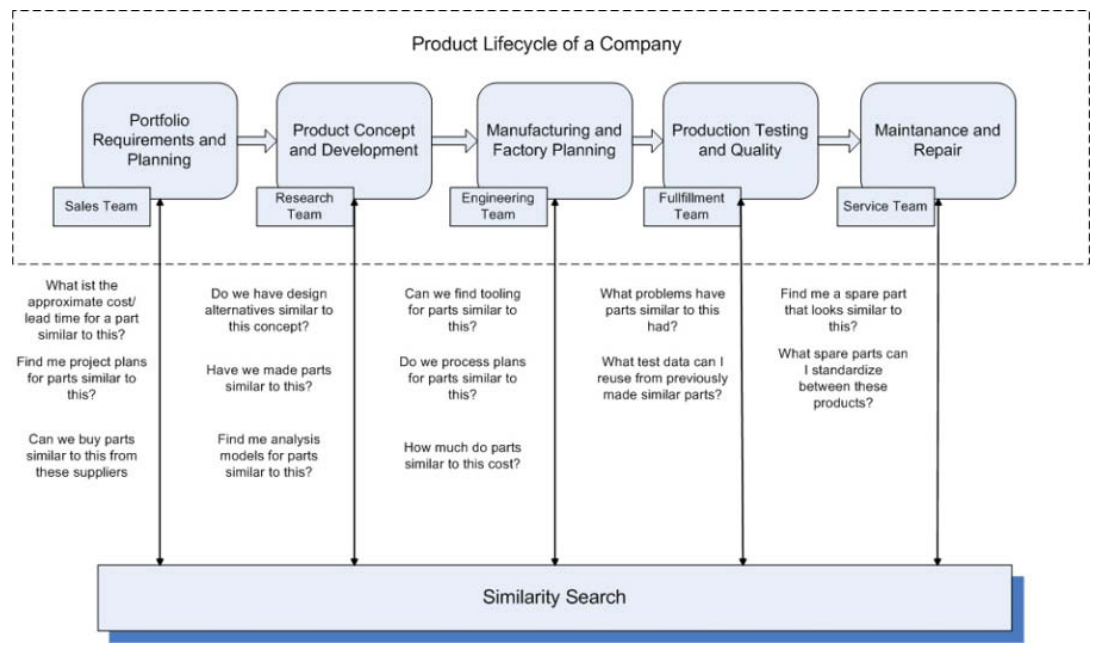

Fig. 1.: Knowledge-reuse across the Product Lifecycle (based on (Iyer 2005))

Collaboration with competitors is very ambivalent. On the one hand system developers cannot handle the entire product. Subcontractors need to be involved to take care of subparts, while the supply chain (or network) becomes larger and more complex. On the other hand there is the company's knowledge representing their most important advantage over their competitors, which has to be protected also in close collaborations with competitors. 
Effective collaboration is only possible if the parties are integrated into one ITsystem to exchange data sufficiently fast and secure. But the two or more parties may be still competitors on other markets, making it inevitable to separate the company's data from the data needed for the work on the mutual project, yet having the possibility of using supporting tools, e.g. a similarity analysis. Another perspective is the combination of the team member's data stores. The questions raised in fig. 1 are still valid, but in a multilateral collaboration there is a broader data pool available which has to be taken into account.

In the following sections the existing solutions and solution approaches will be presented, together with an implementation example of a similarity analysis in the research and development department of an automotive supplier company. This prototype with its underlying process, knowledge and data models was designed and built following the framework.

\section{Reusing Knowledge Based on Similarity Analysis of Product Data}

A similarity analysis of products with the intention of reusing the knowledge hidden in data and associated documents has major advantages. In the first place, the product structure can be reused and modified and this way the time-consuming process of creating the structure of the new product is significantly shortened. Another important aspect is the enhancement in (manufacturing) process reliability. Adapting the specifications from the previous product and avoiding its failures leads to less risk in actual production being extremely valuable for a company. Even "rules of thumb" can be applied when calculating, e.g., the cost-data of the template to estimate reliably approximated costs and present it to the customer in a short time.

The overall goal is the reuse of knowledge from existing documents. To find these documents, a search engine is needed that fulfills the following requirements:

- Diverse data types have to be taken into account, e.g. numerical, alphanumerical, and geometrical (CAD) data, to cover a search over all of the company's relevant documents.

- Search items should be allowed to be fuzzy to achieve first the aspired 'similarity' to exceed e.g. a text search with wildcards and additionally to be able to handle inexact data and information within the process (e.g. resulting from changes in the customer's request over time).

- Integration of the company's heterogeneous data stores into the search engine (e.g. ranging from flat files to ERP or PDM/PLM systems).

- User-, role-, and process-specific interfaces to the search engine (e.g. with context-sensitive selection of valid search items). 
- Access to the search engine and resulting data across a network (via intra/internet)

- Adequate visualization of input and output documents (e.g. 3D visualization of complex geometrical models).

These requirements were implemented as a web application and successfully tested in the research and development department of an automotive supplier. Analyses of the available data sources resulted in three different data types: numerical, alphanumerical, and geometrical (CAD) data. A fuzzy search on numerical data was realized with a threshold of $\pm \mathrm{S} \%$, where $\mathrm{S}$ denotes the maximum percental difference to the search attribute.

The search on alphanumerical data was implemented with the Levenshteinalgorithm (Apostolico 2005), which is applied to determine the so-called "edit distance" between two text attributes. This algorithm computes the minimal number of edit operations necessary to transform one string into another. In relation to the number of characters in the longer string, a distance in the interval $[0,1]$ is generated, which is equivalent to the threshold value $\mathrm{S}$ of the numerical analysis.

Geometrical data in the form of a CAD file is analyzed as follows: The basis for the applied algorithm is not the CAD file itself, but a set of numerical "descriptors" that can be derived from the original file by extracting algorithm-specific properties. Taking into account the time-consuming procedure of building the properties, this is done offline for existing products, and the results are stored in a database. For the similarity analysis of a new CAD file, this file is analyzed online and compared to the already calculated descriptors of existing products. Fig. 2 (left) shows one example user interface of the search engine. In the upper area, the geometry in question is visible. Upload of a geometry file is sufficient for the start of a request. Dimension and position are extracted for orientation reproduced next to the parameter fields in the lower area. The range of the parameters can be widened by applying a threshold in form of a percental value next to the parameter fields. The search is done over all parameters filled with a value. Fig. 2 (right) shows the first two hits from the result list. All search hits are sorted by descending similarity and visualized with selected attributes. Overall, 867 similar parts were found in the data store. The user is now able to select an appropriate part; using the provided key attributes he can gain access to the relevant knowledge documents from the company's data stores. The geometry files in this example are taken from the free 'Engineering Shape Benchmark' of the Purdue University (Purdue 2008). 

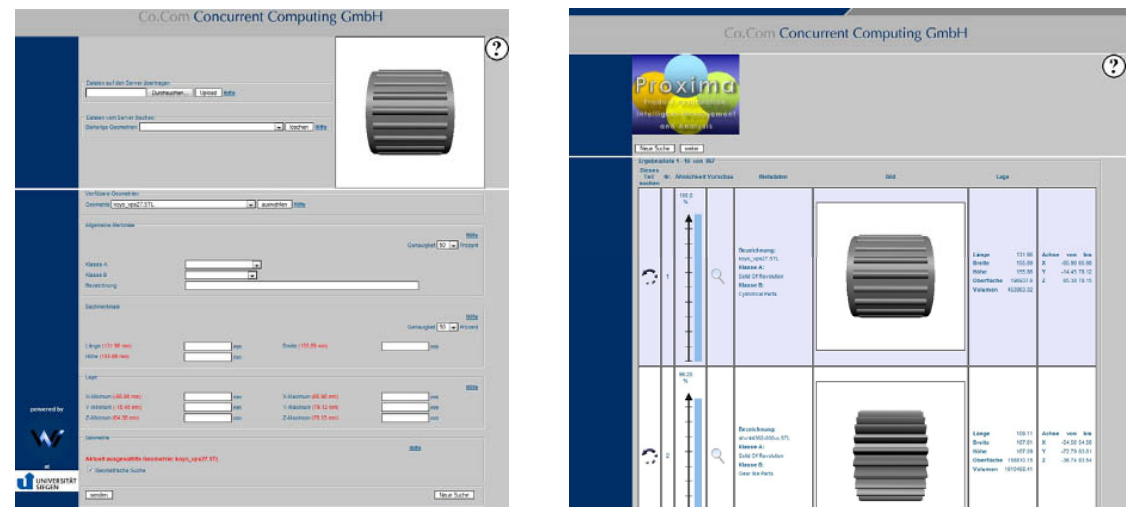

Fig. 2. (left): Example interface to the search engine supporting geometrical search items (uploaded CAD file in the upper right) and other (alpha-)numerical features, e.g. dimension and position. Fig. 2 (right): An example list of search results with similarity measure, file information, visualization of the geometry and additional information, e.g. dimension and position.

\section{Process Support by Reusing Knowledge of Complex Systems}

During the last decade, the global competition and customer's rising requirements forced automotive manufacturers to shorten the lifecycle of their products, thereby reducing the time-to-market while simultaneously being under increasing cost pressure. To reduce cost as well as time-to-market, efficiency not only in production but also in all the previous phase of the product lifecycle must be enhanced. This conflict of objectives is solved by outsourcing not only of the production but also of the design of complex systems to companies of the supplier industry. Complex systems (assemblies represented by hierarchical bills of material, BOM) of this kind consist of up to some hundred parts produced and assembled using various machinery in complex, multi-stage processes (represented by complex working plans) typically in various production sites of one or maybe more collaborating companies. The resulting need for knowledge vastly exceeds the knowledge required for producing rather simple parts. In addition to these problems when designing assemblies, one of the main problems of a system provider (tier-1-supplier) is to keep track of the change requests the manufacturer submits. A supply chain has to be created and the production and actual assembly must be pooled. For every change in one ore more parts of the assemblies, the tier-2supplier and tool manufacturers have to be informed about the design change to keep all the interdependent documents (BOM, working plans, CAD files) synchronized.

The similarity analysis described so far can be used to determine changes in single parts of an assembly and to find similar parts as a template and knowledge 
source for reuse. Even the supply chain can be involved in the process of similarity analysis (see the next chapter for details on this approach).

Similarity analysis of single parts is not sufficient for the analysis of more complex products or a series of different versions of one (complex) product. A system consists not only of parts, but also of relationships and dependencies between them. These have to be taken also into consideration when analyzing such a system. The approach envisaged is to use an ontological model of the automotive domain to represent parts, relations, and dependencies.

Ontology in computer science is a notion for describing and formalizing domain specific concept hierarchies. An ontology provides abstraction from technology, data, or architecture. With an ontology, the semantic correlation of a domain can be represented by using the terms of 'concept' and 'relation', where a concept is the formal representation of a real 'thing' like documents, parts, etc., and 'relation' defines the dependencies between concepts.

Hence, the next step in research is developing an ontological model on the basis of the semantic context of the automotive industry. A product ontology that describes the relationships between different parts in an assembly and all properties and dependencies of a product can be used to measure the similarity between different parts and to filter the search results. Parts that are unequal in main aspects of similarity can be excluded from the search process or deleted from the search results. If a search request is based on one complete product ontology and contains not only single parameters, the measuring process can determine the similarity between different relationships. Just as a complete geometry can be used to optimize the result set it seems promising that a search process that uses semantic data to render the results increases the efficiency of the search.

To be able to use ontology in the search process it is first necessary to design a data model that is able to store all data needed by the ontology and a similarity measure to decide if one product is similar to another. The major problem when using ontology is to gain the relevant data. In most cases the user is required to insert all information into a database or an ontology editor manually. The aim of this ontology-based approach is to develop a method that gains all required information from the existing product data like part lists, work schedules, or cost estimations. Using such a method enables companies to create the desired ontology for each part in the product database automatically.

Having a running prototype that is able to improve the manually search by using numerical, alphanumerical and geometrical data, the next step is to acquire all the information needed to develop a model for the ontology that describes the semantic context of the suppliers and replicates the way experts perceive similarity between complex product structures. Similar to the way the requirements for process support were developed up to now, this can only be achieved by close collaboration with domain experts using prototypical tools reflecting and supporting their usual course of action during the knowledge-intensive processes.

By analyzing the inner structure of a complex system, the dimensions of the parts like length, width, etc., can be extracted with tools of the similarity analysis, 
and can be put in relation to the company's available machine data. That way it is possible to generate an automatic manufacturability analysis. When integrated with a resource management system providing all the relevant technical specifications on the machinery the knowledge structure within an assembly's design and it's working plan (e.g. by matching the physical dimension of a part against the machines dimension) can be exploited to extend the notion of similarity between parts. This extension allows in this context e.g. searching for similarly manufactured products and - based on this - also the generation of valid templates for $\mathrm{BOM}$ and working plans according to a given request. This would further increase a process's efficiency for process participants.

In conjunction with the dependencies inside the system and the knowledge about the capabilities of contractors in the supply chain (see next section), support can be given concerning e.g. where to produce parts or subsystems.

\section{Supporting Knowledge-intensive Processes within Collaborations}

A single company cannot accomplish development and production of complex systems, because different parts and assemblies need to be processed in separate manner. I.e., to weld and finally paint a stamping and a rolling part, four competencies have to be involved (stamping, rolling, welding, and painting). A system developer normally does not have all required competencies available, and therefore has to fall back on the production facilities and competencies of competitors and suppliers.

A project-based collaboration is mostly done in the form of a 'virtual organization' (VO). A VO is a loosely coupled, temporary pool of companies that share resources and skills with the goal of realizing a common interest, e.g. a product or service. In contrast to other organizational forms of cooperation like joint ventures, in a $\mathrm{VO}$ there is no central management level and the parties stay legally independent (Picot et. al. 2003). Communication and coordination is done by the intensive use of IT systems.

If a company creates a supply chain for the development and production of a complex product, the core competencies, skills, knowledge, and resources of the involved participants need to be communicated. For that reason, in addition to the supply chain an 'information chain' has to be set up transferring all relevant data to the appropriate destinations in a two-way-fashion.

To manage these requirements, a 3-layer-model for distributed environments was developed. Fig. 3 shows the concept of the model and the integration of distributed data and knowledge sources. Over the product lifecycle, the knowledge intensive tasks that have to be supported must be identified. In layer 1, 'Process Integration', the similarity analysis is integrated at the appropriate step of the process, where knowledge about previous products is useful and necessary. The 
data sources for the analysis are scattered over the entire supply chain, therefore at layer 2, 'Service Integration', each member of the chain is at least host for one analysis type, geometrical, alphanumerical, or numerical, depending on the data types available. At layer 3, 'Resource Integration', the particular data stores of the hosts have to be integrated into the analysis functionality.

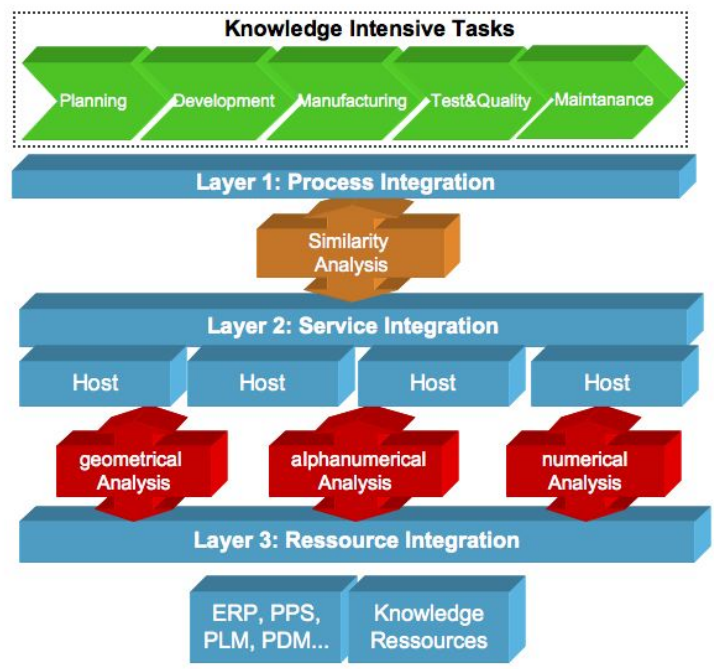

Fig. 3. A three-layer-model for distributed environments supporting knowledge intensive processes

If a participant of the supply chains needs, e.g., information about the producibility of a certain part, he can start the similarity analysis in his company, and feed it with the CAD-model of the part in question. The analysis tool looks up the integrated hosts in the service environment and sends the query to all embedded partners. Depending on the type of query, over the resource integration layer the corresponding database or file base is activated and the query is processed. Results are sent back to the initiator. Every participant has the option of activating or deactivating certain resources for distinct query initiators to handle security issues. Every chain member has to be informed about all other members, because two companies might be both suppliers for a third company, but they are still competitors and therefore might have a special interest of not sharing their skills and knowledge with each other.

Using this architecture, all members of the supply chain are able to look up core competencies, skills, knowledge, and resources of the other participants and speed up their production cycles. 


\section{Conclusions and Future Work}

The subject of this article is to outline a framework for the support of knowledge intensive tasks in manufacturing industries by software tools. Providing the "right" tools requires an understanding of the commonalities between the knowledge-intensive processes to be supported and the process participants and their individual behavior within these processes. As a result, an efficient way of supporting the participants can be derived and adequate tool support conceptualized. In the context of automotive supplier industry, as it is the background of the work presented here, supplying these knowledge workers with documents reflecting the knowledge structure relevant to their reuse in processes was identified as the most promising approach. These documents are found in the data stores of the company on the basis of a similarity analysis. A framework comprising process, knowledge, and data analysis as well as a software architecture for the design and implementation of a similarity analysis tool for searching documents to be reused is validated.

The analysis tools itself was briefly introduced. The search on numerical, alphanumerical, and geometrical data, evaluation and implementation as a web application show the performance and potential benefits of such a search engine.

Beyond the current capabilities of the framework and the search engine, the problem of handling complex products (assemblies) was grasped and the usefulness of the search engine in conjunction with ontology models in this area was addressed. Collaborative environments as a major topic in automotive supplier industry were also introduced and a 3-layer-architecture for widening the search base of the analysis tool over the supply chain for mutual benefit was presented.

Next steps focus on the preparation of an ontology model for automotive supplier industry. Despite the fact that since many years ontology is a matter of academic research and not completely solved, there are promising approaches made in (Maier et al. 2003) especially for automotive supplier industry, that keep track of ontologies as a knowledge representation model. The 3-layer-architecture of the concept for distributed environments has to be implemented to validate the idea.

\section{References}

Apostolico, A. (2005): General Pattern Matching. In: Atallah, A. (ed.): Algorithms and Theory of Computation Handbook. CRC Press 1999, pp. 13-5 - 13-10

Hammer, M., Champy, J. (2001): Reengineering the Corporation. Brealy, London.

Iyer, N., Jayanti, S., Lou, K., Kalyanaraman, Y, Ramani, K. (2005): Shaped-based searching for product lifecycle applications. In: CAD 37.

Jänig, C. (2004): Wissensmanagement. Springer, Berlin.

Lütke Entrup, C, Barth, T., Schäfer, W. (2006): Towards a Process Model for Identifying Knowledge-Related Structures in Product Data. In: Reimer, U., Karagiannis, D. (eds.): Proc. Practical Aspects of Knowledge Management (PAKM 2006), LNAI 4333, pp. 189-200, Springer 
Maier, A., Schnurr, H.P., Sure, Y. (2003): Ontology based Information Integration in the Automotive Industry. In: Proc. of the 2nd Int. Semantic Web Conference (ISWC2003), LNCS 2870, pp. 897-912. Springer.

Riempp, G. (2004): Integrierte Wissensmanagementsysteme. Springer, Berlin.

Purdue Research and Education Center for Information Sciences in Engineering (2008): PRECISE Engineering Shape Benchmark. http://shapelab.ecn.purdue.edu/, (10.01.2008.)

Picot A., Reichwald R., Wiegand R. T. (2003): Die grenzenlose Unternehmung: Information, Organisation und Management, Gabler, Wiesbaden

Remus, U. (2002): Prozessorientiertes Wissensmanagement. Dissertation. Universität Regensburg.

Tietze, Oliver (2003): Strategische Positionierung in der Automobilindustrie. Gabler.

Zelewski, Stephan (2005): Wissensmanagement in Dienstleistungsnetzwerken. Wissenstransfer fördern mit der Relationship Management Balanced Scorecard. DUV, Wiesbaden. 